\title{
Hommages à Pierre-Marie Martin
}

\section{Tributes to Pierre-Marie Martin}

\section{H. Magdelénat · J. Rouessé}

(C) Lavoisier SAS 2019

\section{Hommage à Pierre-Marie Martin par Henri Magdelénat}

\section{Ancien Chef de Départements de l'institut Curie, Paris}

Pierre-Marie Martin, professeur émérite de la faculté de médecine d'Aix-Marseille, nous a quittés brutalement un soir de février 2019, à l'âge de 76 ans.

Pour ses pairs, ses collaborateurs et ses élèves, il laissera en héritage une œuvre scientifique riche et originale et le souvenir d'une forte personnalité. Car, scientifiquement, c'était un... perturbateur (endocrinien, assurément !), refusant les idées toutes faites et les pressions de toutes sortes, ce qui lui a valu beaucoup de... disons, contrariétés. Mais heureusement aussi, de nombreux et vrais amis. Au retour, dans les années 1980, de son séjour dans le laboratoire de Bill McGuire aux États-Unis, où ont germé déjà ses idées sur les perturbateurs endocriniens, sur l'intérêt des récepteurs stéroïdiens pour le traitement ciblé des cancers du sein, sur l'importance des banques de tumeurs pour la recherche biomédicale, il a créé puis développé, d'abord dans son laboratoire à Marseille, ensuite au sein de l'EORTC, la discipline de biopathologie. Il y a consacré toute son énergie et dans bien des domaines, ce fut un pionnier.

Mais bien davantage que le professeur et sa carrière scientifique, pour ses proches et pour ses amis, c'était Pierre, avec son immense générosité émotionnelle. Il aimait ses amis et remuait des montagnes pour leur venir en aide. Il aimait, en véritable esthète, les belles choses, les artisans authentiques, la bonne cuisine et les grands vins, les livres, la musique, baroque surtout, la montagne (il fut guide dans les Écrins), le chalet de Corps qu'il avait construit avec son père et sa ville d'adoption, Marseille. Il aimait les animaux, les chiens, les chats et même les tortues, dont il s'entourait.

Avec Pierre, nous perdons cette part de connaissances, de culture et de civilisation qu'il nous faisait partager. Comme sa famille à laquelle nous pensons, nous, ses amis, ne devons pas l'oublier.

\section{Éloge du Pr Pierre-Marie Martin 1943-2019 par le Pr Jacques Rouessé}

\author{
Membre de l'Académie nationale de médecine, ancien \\ secrétaire général de la SFSPM \\ Au nom de la SFSPM — Président : Dr Bruno Cutuli
}

C'est en tant qu'ami clinicien que je me permets, au nom de la Société française de sénologie et de pathologie mammaire (SFSPM), ce bref éloge de Pierre-Marie Martin. PierreMarie vient de nous jouer un tour à sa façon en nous quittant sans nous prévenir, ce qui est bien dans le style de ce talentueux et souvent visionnaire électron libre.

Dès le début de sa carrière, il nous surprend : quittant rapidement la chirurgie, il embrasse la biologie, lui apportant la rigueur de l'opérateur liée à celle du raisonnement. Son titre de chef de service du laboratoire de transfert d'oncologie biologique résume bien toute son activité. Son œuvre est un modèle de recherche translationelle, de la paillasse au soin. Professeur de classe exceptionnelle de l'université AixMarseille, enseignant et chercheur, il dirige à l'AP-HM de nombreuses équipes de biopathologie tissulaire. Son épreuve de « Titres et travaux » est impressionnante, 423 publications indexées Pubmed en témoignent de même que les différents prix nationaux et internationaux dont il est honoré.

Son nom, avec celui de quelques autres Français, est tout d'abord attaché aux récepteurs hormonaux du cancer du sein. Son séjour de près de deux ans (1977-1979) à San Antonio, chez le grand maître du sujet William McGuire, cautionne la validité de ses travaux. Il s'impliquera à la mise en place des contrôles de qualité de leur évaluation à un niveau national et européen. Cette thématique l'amène tout naturellement à être en 1977 l'un des fondateurs de notre société, la SFSPM, dont il restera à ce titre membre du CA. Mais Pierre-Marie ne s'intéresse pas au seul cancer du sein, il travaille sur celui de la prostate, le méningiome, le gliome, les tumeurs colorectales, celles de la vessie et de façon générale sur le microenvironnement tumoral (le fameux stroma !!), autant de domaines auxquels il se consacre avec 
ardeur et compétence. Un de ses thèmes favoris est la recherche de témoins de l'agressivité tumorale tels uPA, PAI-1 et leur valeur pronostique. Bien entendu, cette riche activité de recherche lui vaut une reconnaissance internationale, non seulement comme membre de nombreuses sociétés savantes, mais surtout comme acteur majeur de groupes de travail.

Au-delà de cette recherche au laboratoire, la formation médicale de Pierre-Marie l'a profondément marqué comme peuvent l'attester tous les cliniciens qui le rencontraient, appréciant son sens pratique, très concret et sa grande rigueur intellectuelle. Profondément original, d'une grande culture non seulement biologique mais aussi générale, enthousiaste, parfois indigné, d'une foncière intégrité lui interdisant toute compromission, nous perdons avec lui un collègue exceptionnel, un grand scientifique et surtout un ami irremplaçable. 